\title{
A PESSOA COM AVC EM PROCESSO DE REABILITAÇÃO: GANHOS COM A INTERVENÇÃO DOS ENFERMEIROS DE REABILITAÇÃO
}

\section{THE PERSON WITH STROKE IN REHABILITATION PROCESS: GAINS FROM THE INTERVENTION OF REHABILITATION NURSES LA PERSONA CON ACCIDENTE CEREBROVASCULAR EN EL PROCESO DE REHABILITACIÓN: GANANCIAS DE LA INTERVENCIÓN DE ENFERMERAS DE REHABILITACIÓN}

DOI 10.33194/rper.2020.v3.n2.6.5799 | Submetido 01/08/2020 | Aprovado 15/12/2020

\author{
Jéssica Teixeira Santos ${ }^{1}$; ;élia Maria Silva Campos $^{1}$ (D); Maria Manuela Martins ${ }^{2}$ \\ 1 - Centro Hospitalar e Universitário do Porto; 2 - Escola Superior de Enfermagem do Porto
}

\section{RESUMO}

Introdução: As Doenças Cérebro Vasculares constituem uma das principais causas de morte e de morbilidade em Portugal, causando incapacidade e invalidez.

Objetivo: Analisar os ganhos em saúde de um doente com AVC após implementação de um Programa de Reabilitação. Método: Estudo de caso de um doente que sofreu um AVC, de natureza descritiva, num serviço de internamento de um Hospital do Norte Litoral do país, durante 30 dias.

Resultados: Após implementação do Programa de Reabilitação verificou-se uma melhoria no equilíbrio corporal, força muscular, capacidade para o autocuidado e deglutição e diminuição do risco de queda.

Conclusão: A intervenção do Enfermeiro Especialista em Enfermagem de Reabilitação traz ganhos na recuperação funcional do doente com AVC, contribuindo para a sua autonomia nas atividades de vida diária.

Palavras-chave: acidente vascular cerebral; reabilitação; autocuidado; ganhos em saúde; enfermagem

\section{RESUMEN}

Introducción: Las enfermedades cerebrovasculares son una de las principales causas de muerte y morbilidad en Portugal, causando discapacidad e invalidez.

Objetivo: Analizar las ganancias de salud de un paciente con accidente cerebrovascular después de implementar un programa de rehabilitación.

Método: Estudio de caso de un paciente que sufrió un derrame cerebral, de naturaleza descriptiva, en un servicio de hospitalización de un hospital en la costa norte del país, durante 30 días.

Resultados: Después de la implementación del Programa de Rehabilitación hubo una mejora en el equilibrio corporal, la fuerza muscular, la capacidad de autocuidado y la deglución y la disminución del riesgo de caída.

Conclusión: La intervención de la Enfermera Especialista en Enfermería de Rehabilitación trae ganancias en la recuperación funcional de los pacientes con accidente cerebrovascular, lo que contribuye a su autonomía en las actividades de la vida diaria.

Palabras clave: accidente cerebrovascular; rehabilitación; autocuidado; beneficios de salud; enfermería

\section{ABSTRACT}

Introduction: Cerebrovascular diseases are one of the main causes of death and morbidity in Portugal, causing disability and invalidity.

Objective: To analyze the health gains of a stroke patient after implementing a Rehabilitation Program.

Method: Case study of a patient who suffered a stroke, of a descriptive nature, in an inpatient service of a North Coast Hospital in the country, for 30 days.

Results: After the implementation of the Rehabilitation Program, there was an improvement in body balance, muscle strength, capacity for self-care and swallowing and decreased risk of falling.

Conclusion: The intervention of the Rehabilitation Nurse brings gains in the functional recovery of stroke patients, contributing to their autonomy in activities of daily living.

Keywords: stroke; rehabilitation; self-care; health gains; nursing 


\section{INTRODUÇÃO}

A Enfermagem de Reabilitação reúne um conjunto de competências específicas, de caráter multidisciplinar, que têm por base conhecimentos e procedimentos que permitem cuidar das pessoas com doenças agudas, crónicas ou com as suas sequelas com o intuito de retomar a sua máxima funcionalidade $e$ independência. ${ }^{(1)}$

Assim, é função do Enfermeiro de Reabilitação assistir no processo de recuperação do doente com vista á sua máxima autonomia funcional, mas também no sentido de manter a sua autoestima e qualidade de vida, contribuindo para o seu desenvolvimento pessoal. (1)

Uma das razões pelas quais nos faz sentido ser um enfermeiro, particularmente da área da reabilitação, a intervir neste tipo de situações é fundamentado por Virgínia Henderson (1966) que refere que “(...) a única função da enfermeira é a de assistir o indivíduo, doente ou saudável, no desempenho das atividades que contribuem para a saúde ou para sua recuperação (...) que executaria sem auxílio caso tivesse a força, a vontade e os conhecimentos necessários. E fazê-lo de modo a ajudá-lo a conseguir a independência tão rapidamente quanto possível."(2) Cabe, portanto, ao enfermeiro intervir no sentido de despertar no doente esta força, quer física quer psicológica, dotando-o também dos conhecimentos necessários, para que este possa retomar o mais rapidamente possível uma vida autónoma.

É neste sentido que o Enfermeiro Especialista em Enfermagem de Reabilitação deve ser capaz de elaborar e implementar um programa de reabilitação, que se baseia um conjunto de intervenções em que estão programadas determinadas rotinas de exercícios, que devem ser implementados de modo progressivo e individualizado, tendo em conta as capacidades de cada doente, com o objetivo de promover a sua funcionalidade. ${ }^{(4)}$

As doenças cardiovasculares, em particular a Doença Isquémica Cardíaca e as Doenças Cérebro Vasculares, constituem a principal causa de morte em Portugal $(29,7 \%)$, sendo também uma das principais causas de morbilidade, incapacidade e invalidez, privando precocemente as pessoas de anos de produtividade e de qualidade de vida. ${ }^{(4)}$

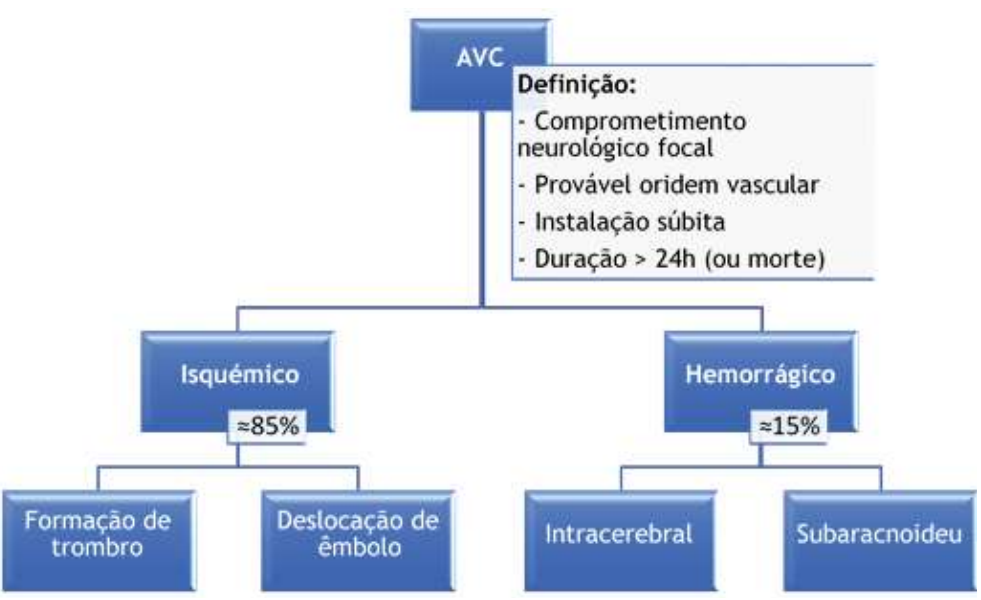

Figura 1 - Síntese da patogenia do AVC, adaptado de Organização Mundial da Saúde (5)
Temos vindo a assistir a um envelhecimento progressivo da população, o que tem levado a uma tendência crescente do número de internamentos por doenças do foro circulatório, tendo-se registado um aumento de $1,6 \%$ de internamentos por AVC entre 2011 e 2016, sendo que em 2016 o número total de internamentos de doentes vítimas de AVC ultrapassava os 25.000, com uma taxa de mortalidade de $49,7 \% .{ }^{(4)}$

0 AVC define-se como um comprometimento neurológico focal ou global, de provável origem vascular, que ocorre de forma súbita e tem duração superior a 24 horas, podendo mesmo causar a morte. ${ }^{(5)}$ Pode ser de origem isquémica, caraterizado por uma oclusão súbita de artérias que irrigam o cérebro, devido a um trombo localmente formado ou por deslocação de um êmbolo; ou então de causa hemorrágica, classificando-se como intracerebral, se ocorrer no tecido cerebral, ou subaracnoideia, se surgir entre as duas meninges conhecidas como pia-máter e aracnoide. (5)

Um episódio de AVC tem habitualmente como consequência sinais/sintomas de compromisso neurológico, como diminuição da força muscular, descoordenação, alterações da linguagem (afasia motora e/ou sensorial), comprometimento da visão a nível campimétrico (hemianopsia) ou desvio do olhar conjugado, apraxia, ataxia, disartria, disfagia, podendo mesmo afetar a função cognitiva e o estado de consciência. ${ }^{(5)}$

Estes défices levam geralmente a compromissos na marcha, no controlo postural e no equilíbrio, limitando a mobilidade e a capacidade para executar as atividades de vida diária e aumentando também o risco de queda. (6)

Assim, torna-se essencial a intervenção do Enfermeiro Especialista em Enfermagem de Reabilitação, que vai assistir na reeducação das funções motoras, sensoriais e/ou cognitivas perdidas. ${ }^{(1)}$

Em Portugal, o Enfermeiro de Reabilitação orienta habitualmente a sua intervenção a partir dos princípios teóricos da Margaret Johnstone, que defende uma reabilitação baseada na prevenção da espasticidade, através de posicionamentos no leito com padrão antiespástico; e na recuperação de défices do lado lesado, através de exercícios de reeducação sensoriomotora, tendo por base as fases do desenvolvimento motor do recém-nascido humano (fase de lactente). ${ }^{(7)}$

Esta corrente teórica privilegia o início das atividades no leito, através das técnicas de posicionamento antiespástico, que devem ser implementadas durante as $24 \mathrm{~h}$ logo na fase aguda, e também exercícios de automobilização, seguindo-se o rolar, a quadripedia, o sentar e, por último, a marcha. Estes são os pontos de referência da teoria de Margaret Johnstone que, apesar de antiga, tem-se demonstrado consistente nos ganhos em saúde para o doente após um evento tão incapacitante como o AVC. (7)

A recuperação neurológica é mais significativa nos três a seis meses após o AVC, sendo as primeiras semanas cruciais para o progresso na recuperação, pois é quando 
se observam os principais sinais de melhoria se o doente for devidamente estimulado e reabilitado. ${ }^{(8)}$

Posto isto, é importante iniciar o programa de reabilitação o mais precocemente possível, ou seja, assim que a pessoa esteja hemodinamicamente estável. Para uma recuperação de sucesso, este deve ser um programa contínuo ao longo do tempo e deve existir cooperação e coordenação multidisciplinar entre todos os elementos da equipa de profissionais de saúde. ${ }^{(3)}$

Pretendemos demonstrar, com base no relato de um caso clínico em particular, quais os ganhos em saúde que resultam da intervenção do Enfermeiro Especialista em Enfermagem de Reabilitação na fase aguda após um episódio de AVC.

\section{METODOLOGIA}

Trata-se de um Estudo de Caso de natureza descritiva, partindo-se de um fenómeno contemporâneo da vida real, e cuja questão de investigação responde às questões “Como?” (com a Intervenção dos Enfermeiros de Reabilitação) e "Porquê?" (para obter ganhos na pessoa com AVC em processo de reabilitação). ${ }^{(9)}$

Iniciámos por propor ao doente a possibilidade de participar no estudo de caso, tendo sido elaborado posteriormente o planeamento dos cuidados de acordo com o modelo em uso no serviço, que contempla a fase de avaliação inicial, planeamento de cuidados, intervenção dos Enfermeiros Especialistas em Enfermagem de Reabilitação e, por último, a avaliação final. Para garantir os princípios éticos, no primeiro momento foi realizado o consentimento informado por escrito.

Uma das estratégias usadas para colheita de dados centrou-se na aplicação de Escalas, entre elas:

- Índice de Tinetti: avalia o equilíbrio em posição de sentado e em posição ortostática. ${ }^{(10)}$ Foi criada em 1986 por Tinetti, Williams e Mayewski e o seu score indica alto risco de queda se valores $\leq 18$, moderado risco de queda se valores entre 19 - 23 e baixo risco de queda se valores $\geq 24$. $^{(11)}$
- Escala de Morse: criada por Janice Morse, tendo surgido pela primeira vez em 1985 e posteriormente passou por várias fases de reconstrução até à ultima versão conhecida em 1989. O score final indica o risco de queda, considerando-se alto risco de queda quando o resultado é $\geq 45$ pontos. ${ }^{(12)}$

- Medical Research Council Muscle Scale: elaborada pelo Conselho de Pesquisa Médica do Reino Unido (Medical Research Council), em 1943. Avalia a força muscular dos membros superiores e inferiores, variando entre grau 0 (sem contração muscular palpável ou visível) e grau 5 (força normal). ${ }^{(13)}$

- Gugging Swallowing screen (GUSS): avaliação da deglutição/despiste de disfagia, normalmente aplicado em pessoas após AVC, embora também possa ser usada noutros contextos; também auxilia na decisão da dieta recomendada conforme o grau de disfagia. Foi criada por Michaela Trapl, Paul Enderle, Monika Nowotny, Yvonne Teuschl, Karl Matz, Alexandra Dachenhausen e Michael Brainin em 2007. É composta por 2 fases: Avaliação Indireta e Avaliação Direta. Se o Score total for 20 indica Sem Disfagia, 15 - 19 Disfagia Ligeira, 1014 Disfagia Moderada e 0-9 Disfagia Grave. ${ }^{(13)}$

- Escala de House-Brackmann: classificação do nível de lesão nervosa numa paralisia facial. Foi criada por House JW e Brackmann, em 1985, e varia entre Grau I (Face normal) e Grau VI (Paralisia total). ${ }^{(13,14)}$

- Índice de Barthel: avalia o nível de dependência para a realização de dez atividades de vida diária: alimentarse, higiene pessoal, uso do WC, tomar banho, vestir e despir, controlo de esfíncteres (intestinal e urinário), andar/mobilidade, transferir-se, subir e descer escadas. Os autores da escala são Mahoney FI e Barthel D (1965) e varia de 0-100, em que 0-20 indica Dependência total; 20-35 Dependência severa; 40-55 Dependência moderada; 60-89 Dependência ligeira; 90 100 Independência. $^{(13,15)}$

Seguiu-se o planeamento dos cuidados sustentado num programa de intervenção, que foi constituído por três fases: Fase 1 - exercícios deitado, Fase 2 - exercícios sentado e Fase 3 - exercícios em posição ortostática; tendo por base o programa habitual instituído no serviço para reabilitação de uma pessoa após AVC.

Fase 1 (Exercícios deitado)
- Mobilização passiva do hemicorpo
afetado, em padrão anti-espástico (10x
cada articulação)
- Automobilização vertical e cruzada dos
membros superiores (10x)
- Controlo do joelho com bola (10x)
- Ponte (10x)
- Dissociação de cinturas (escapular e
pélvica) (10x)
- Rolar (10x) cada carga no
- Facilitação cruzada com carga
cotovelo (10x)
- Exercícios orofaciais (10x cada
exercício)

Fase 2 (Exercícios sentado)
- Manter postura do tronco (sem apoio)
(5 min)
- Causar desiquilíbrio multidirecional
(10x)
- Flexão/extensão do cotovelo (halteres
0,5 kg) (10x)
-Adução/abdução de braços (banda
elástica) (10x)
- Flexão/extensão do joelho e quadril
(banda elástica) (10x)
- Pedalar (pedaleira motorizada) (2kg, 20
min, 18 rpm)
- Exercícios orofaciais (10x cada
exercício)

Figura 2 - Programa de Exercícios de Reabilitação após AVC 

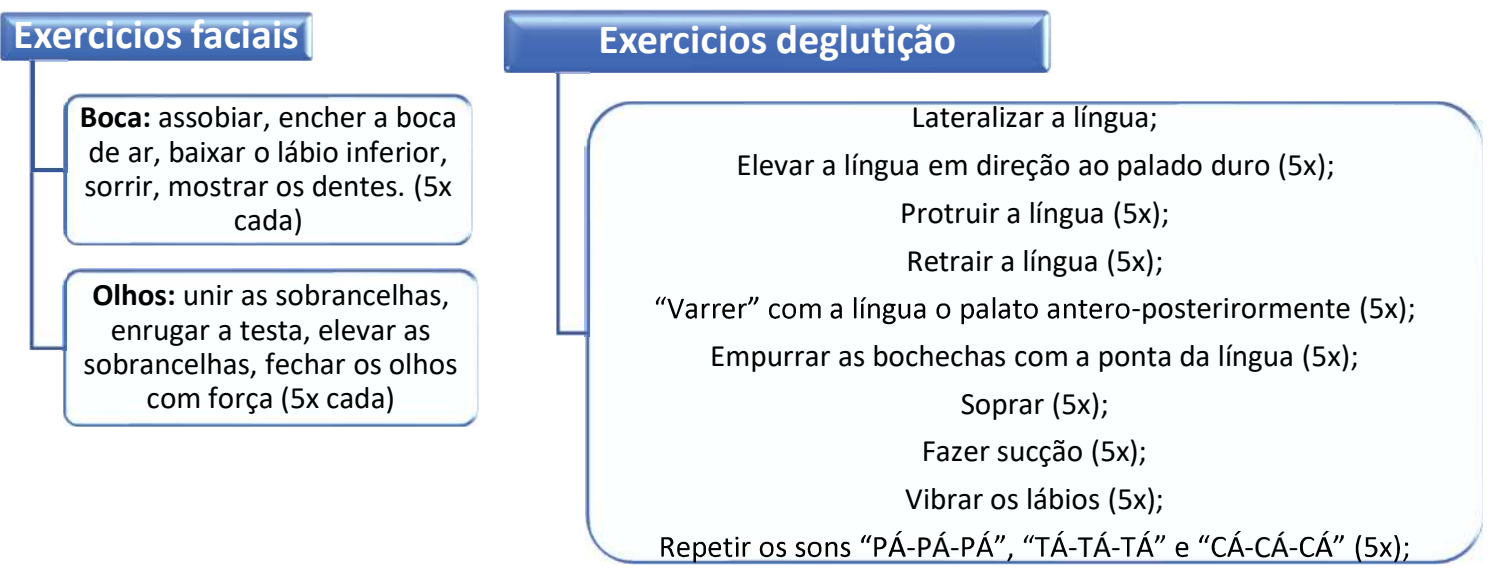

Figura 3 - Programa de Exercícios de Orofaciais

Importa referir que este foi o único programa de reabilitação ao qual o doente foi submetido, não existindo outras terapias em paralelo relacionadas com a recuperação funcional do doente. O programa de exercícios foi implementado conforme o previsto, todos os dias, no turno da manhã, exceto ao sábado e domingo. A Fase 1 foi implementada entre os dias D1 e D3, os exercícios da Fase 2 iniciaram-se no D2 e a Fase 3 foi implementada a partir de D3.

\section{RESULTADOS}

A assistência ao doente iniciou-se no dia 30/6/2020 (D1) com a avaliação inicial de acordo com as normas de orientação do hospital e com a aplicação das escalas previstas na metodologia.

\section{Avaliação inicial}

A Avaliação Inicial consiste na primeira fase do Processo de Enfermagem, em que há uma recolha organizada e sistemática de dados relevantes para identificar os focos de atenção que permitem elaborar os Diagnósticos de Enfermagem. Esta informação também ajuda a conhecer os recursos familiares e/ou comunitários do doente e possibilita uma planificação de intervenções que sejam adequadas às suas necessidades e particularidades. ${ }^{(16)}$

O doente em estudo tem 76 anos (M.G.), é do sexo masculino, caucasiano, reformado $\left(5^{\circ}\right.$ ano de escolaridade) e sofreu um AVC Hemorrágico a 24/06/2020, de etiologia hipertensiva. Segundo TC cerebral na admissão hospitalar, apresentava hemorragia intra-axial aguda no tálamo esquerdo, com dois componentes: um mais dorsomedial, a partir do qual a hemorragia se estendeu para os ventrículos supratentoriais, e outro mais póstero-superior, tálamocapsular, sendo que no seu conjunto apresentam cerca de $28 \mathrm{~mm}$ e $30 \mathrm{~mm}$ de maiores eixos, respetivamente, nos planos coronal e axial.

O Sr. M.G. tem como antecedentes médicos relevantes Hipertensão arterial, Diabetes, Obesidade, Cardiopatia hipertensiva, Patologia osteoarticular degenerativa (já deambulava com uma canadiana), Prótese Total do Joelho bilateral (esquerda em fevereiro 2018, direita em março 2019) e Discopatia L4-L5, já submetido a cirurgia. A medicação domiciliária habitual é: Pantoprazol 20mg (jejum), Ramipril 5mg e Bisoprolol $5 \mathrm{mg}$ (pequeno-almoço), Atorvastatina 20mg e Amlodipina 10mg (jantar) e Trazodona 50mg (ceia).

Por apresentar peso corporal de $117 \mathrm{Kg}$ e 1,72 m de altura, encontra-se com quadro de Obesidade Grau II (Figura 4).

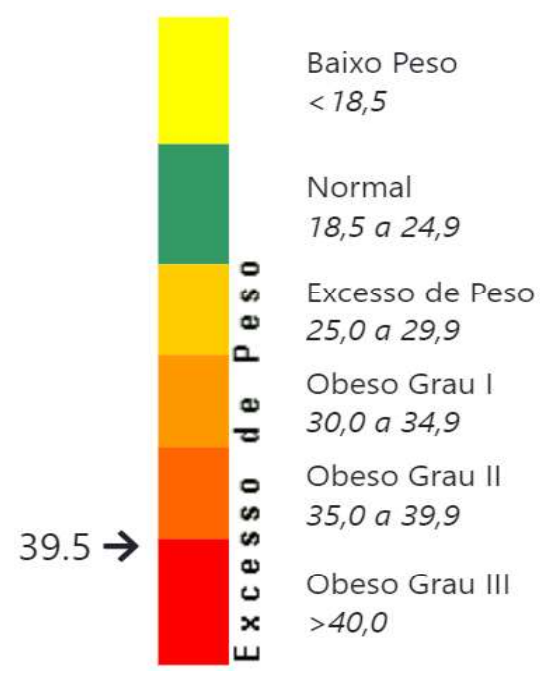

Figura 4 - Tabela de IMC (adaptado de La Roche ${ }^{17}$ )

Deu entrada na Urgência no dia em que ocorreu o AVC, tendo passado para a Unidade de Cuidados Intermédios de Medicina (UCIM) para controlo tensional, devido ao quadro de emergência hipertensiva, e foi admitido a 26/06/2002 no serviço de Neurologia de um Hospital da Região do Norte Litoral do país.

No internamento, manteve-se hemodinamicamente estável, sem alterações do estado de consciência (Score 15 na Escala de Coma de Glasgow) e sem compromisso da função cognitiva (Score de 27 na Escala de MiniMental State Examination, indicando Sem Défices Cognitivos num doente que tenha entre 1 e 11 anos de escolaridade).

À entrada no serviço apresentava como défices neurológicos: hemiparesia à direita (face, membro superior e membro inferior); alterações do equilíbrio estático e dinâmico em posição de sentado (sem apoio lateral) e em posição ortostática; disfagia para líquidos e disartria. Consequentemente, apresentava limitações na mobilidade e na capacidade para executar as suas atividades de vida diária. 
Vivia com a esposa, a filha (Sra S.) e a neta de 18 anos. Quando a Sra S. foi visitar o pai, referiu à equipa médica e de enfermagem que apenas poderia levar o doente para casa quando estivesse praticamente autónomo, pois a sua mãe já estaria dependente de cuidados e não teria disponibilidade para cuidar dos dois. O Sr.M.G. era autónomo nas atividades de vida diárias antes do AVC, embora já com limitações devido à patologia osteoarticular. Já deambulava com uma canadiana e tinha estratégias adaptativas para algumas atividades de autocuidado, como sentar-se para tomar banho, vestir e despir os membros inferiores e calçar e descalçar os sapatos.

\section{Fase de desenvolvimento}

Com base na informação clínica do doente, foi possível identificar um conjunto de diagnósticos e intervenções de enfermagem de reabilitação (Figura 5), com recurso à Classificação Internacional para a Prática de Enfermagem (CIPE $®)$ versão 2019. ${ }^{(18)}$

Pode ver-se no Gráfico 1 que se foi verificando uma recuperação do equilíbrio corporal do doente durante a implementação do Programa de Exercícios de Reabilitação.

Entre D1 e D3 não se verificaram alterações do equilíbrio corporal do Sr.M.G., no entanto, entre D3 e D9 houve melhorias no equilíbrio estático, coincidindo com os dias em que se aplicaram maioritariamente exercícios da Fase 2 do Programa de Reabilitação, que inclui o treino de força muscular dos membros superiores e equilíbrio do tronco na posição de sentado.

A partir de D15 é quando mais se verificam melhorias no equilíbrio dinâmico do doente, começando a evidenciar-se mais progressos no treino de marcha.

\begin{tabular}{|c|c|c|c|}
\hline $\begin{array}{l}\text { Diagnósticos de } \\
\text { Enfermagem }\end{array}$ & Intervenções & $\begin{array}{l}\text { Data de } \\
\text { início }\end{array}$ & $\begin{array}{l}\text { Data de } \\
\text { fim }\end{array}$ \\
\hline $\begin{array}{l}\text { Movimento corporal } \\
\text { diminuído à direita }\end{array}$ & $\begin{array}{l}\text { - Executar exercícios musculares e articulares passivos }(1 \mathrm{x} / \text { dia }) \\
\text { - Ensinar/Instruir/Treinar exercícios musculares e articulares }(1 \mathrm{x} / \mathrm{dia})\end{array}$ & $\begin{array}{c}30 / 06 \\
\text { (D1) }\end{array}$ & $\begin{array}{l}29 / 07 \\
(\mathrm{D} 30)\end{array}$ \\
\hline $\begin{array}{l}\text { Paresia da Face à } \\
\text { Direita }\end{array}$ & $\begin{array}{l}\text { - Executar massagem da face à direita }(1 \mathrm{x} / \text { dia }) \\
\text { - Ensinar / Instruir / Treinar exercícios da face }(1 \mathrm{x} / \text { dia })\end{array}$ & $\begin{array}{l}30 / 06 \\
\text { (D1) }\end{array}$ & $\begin{array}{l}8 / 07 \\
(D 9)\end{array}$ \\
\hline $\begin{array}{l}\text { Equilibrio Corporal } \\
\text { Comprometido } \\
\end{array}$ & $\begin{array}{l}\text { - Ensinar/Instruir/Treinar técnica de exercícios de equilíbrio corporal } \\
\text { (1x/dia) }\end{array}$ & $\begin{array}{c}30 / 06 \\
(\mathrm{D} 1)\end{array}$ & $\begin{array}{l}29 / 07 \\
(D 30)\end{array}$ \\
\hline $\begin{array}{l}\text { Deglutição } \\
\text { Comprometida }\end{array}$ & $\begin{array}{l}\text { - Otimizar posicionamento à refeição }(9 \mathrm{~h}, 12,16 \mathrm{~h}, 19 \mathrm{~h}, 22 \mathrm{~h}) \\
\text { - Vigiar a refeição }(9 \mathrm{~h}, 12,16 \mathrm{~h}, 19 \mathrm{~h}, 22 \mathrm{~h}) \\
\text { - Ensinar/Instruir/Treinar técnica de exercícios de para deglutição } \\
\text { (1x/dia) }\end{array}$ & $\begin{array}{c}30 / 06 \\
\text { (D1) }\end{array}$ & $\begin{array}{l}20 / 07 \\
(D 21)\end{array}$ \\
\hline $\begin{array}{l}\text { Capacidade para o } \\
\text { Autocuidado } \\
\text { Comprometida }\end{array}$ & $\begin{array}{l}\text { - Assistir no Autocuidado (Sem horário) } \\
\text { - Incentivar a executar as atividades de autocuidado (Sem horário) }\end{array}$ & $\begin{array}{c}30 / 06 \\
(\mathrm{D} 1)\end{array}$ & $\begin{array}{l}29 / 07 \\
\text { (D30) }\end{array}$ \\
\hline
\end{tabular}

Figura 5 - Diagnósticos e Intervenções de Enfermagem de Reabilitação

O Sr.M.G. inicou treino de marcha com andarilho a partir de D4, inicialmente com um padrão de marcha hesitante e passos assimétricos. Entretanto, verificouse uma marcha mais segura no D15, por isso progrediuse nesse dia para treino de marcha com canadianas, com melhorias significativas do equilíbrio dinâmico. No D17 iniciou-se o treino de marcha com canadianas nas escadas e a partir do D23 o doente já só usa uma canadiana para andar, tal como antes do AVC.

\section{Equilíbrio, Autocuidado e Queda}

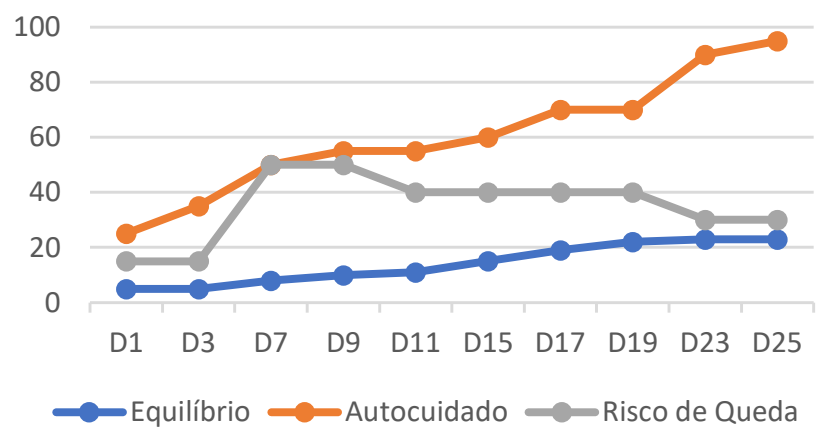

Gráfico 1 - Ganhos no Equilíbrio, Autocuidado e Queda
Note-se que entre D1 e D3 obtinha apenas 15 pontos na Escala de Morse (baixo risco de queda), uma vez que nesses dias o doente ainda se mantinha no cadeirão, mas no D7 já apresentava 50 pontos no score da escala de Morse (alto risco de queda).

Entre D3 e D7 há um aumento significativo da pontuação do Índice de Barthel, marcando a transição para a independência nos items Alimentar-se e Higiene Pessoal (barbear, dentes, rosto e cabelo), que coincidiu com a melhoria do equilíbrio do Sr.M.G em posição de sentado, o que the permitiu retomar as tarefas que podia executar sentado na cadeira-sanita ou no cadeirão.

No D9, o Sr.M.G já conseguia executar as Transferências; o Controlo Urinário foi retomado no D15; e entre D15 e D17 verifica-se novamente um acréscimo no seu nível de autonomia, desta vez pelo facto de ter passado a ser capaz de Subir e Descer Escadas com canadianas a partir de D17. Por fim, verifica-se um progresso acentuado no Autocuidado entre D19 e D23, que coincide com a fase em que o doente se tornou autónomo no Uso do Sanitário e no Tomar Banho, tratando-se, assim, do ponto de cut-off (momento de transição da dependência para a 
independência). Entre D23 e D25 acrescem ainda 5 pontos na Escala de Barthel, relativos às últimas melhorias evidenciadas pelo doente no culminar deste processo de Reabilitação, nomeadamente no item da Mobilidade.

Foi-se verificando, portanto, uma melhoria gradual no nível de independência do Sr.M.G., que culminou com a sua autonomia em todas as atividades de autocuidado com recurso a apenas uma canadiana para andar e subir e descer escadas, tendo deste modo tido alta na mesma situação de autonomia em que se encontrava anteriormente ao AVC.

0 controlo urinário foi pontuado com 0 até D11, pois neste período o doente esteve algaliado devido a episódios de retenção urinária, consequentes de um processo de infeção urinária.

Além do equilíbrio corporal, também a Força Muscular no hemicorpo afetado foi aumentando. Segundo a Medical Research Council Muscle Scale, de D1 a D17 o doente apresentava força muscular do membro superior direito de grau 4 e no D25 já apresentava grau 5; no membro inferior direito evoluiu de grau 3 para grau 4 ao longo do processo de reabilitação.

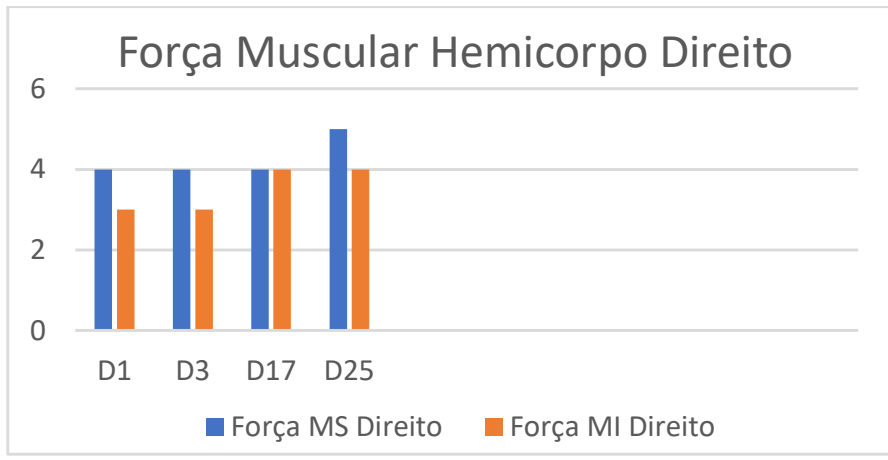

Gráfico 2 - Ganhos na Força Muscular

A Escala de Guss foi aplicada de 5/5 dias revelando disfagia para líquidos (13 pontos: grau moderado) nos dias D1 e D6, altura em que o doente se alimentava de dieta pastosa e era hidratado com água gelificada. No D11 apresentava ainda deglutição demorada para sólidos (19 pontos), apresentando apenas disfagia ligeira e por isso já se alimentava de dieta geral mole. No D21 já deglutia todos os alimentos com sucesso.

NaEscala de House-Brackmann, apresentava paresia facial à direita de Grau III (disfunção moderada) entre D1 - D7, tendo posteriormente evoluído para Grau II (disfunção ligeira) que manteve até ao final da intervenção.

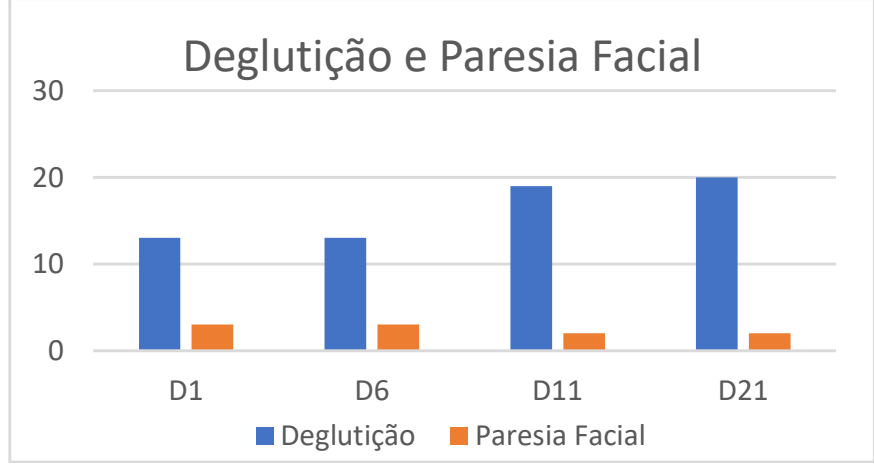

Gráfico 3 - Ganhos nos Músculos Orofaciais
Após a fases de colheita e análise de dados, segue-se a discussão de resultados, através de uma reflexão sobre os mesmos e comparação com outros estudos.

\section{DISCUSSÃO}

Através da análise dos resultados apresentados, depreende-se que existiu em todos os indicadores uma tendência crescente, tendo-se verificado ganhos em saúde.

O equilíbrio corporal teve uma melhoria gradual, principalmente a partir do D15, e a força muscular do lado parético foi outro indicador que melhorou à medida que os exercícios de reabilitação íam sendo executados pelo doente. Estes dois indicadores são extremamente importantes, quer para iniciar a marcha, quer para a capacidade de desempenhar tarefas funcionais, permitindo que o doente readquira a sua autonomia. ${ }^{(20)}$

O Sr.M.G evoluiu com sucesso no treino de marcha, tendo conseguido progredir do andarilho para canadianas e, posteriormente, para o uso de apenas uma canadiana. Estes progressos também se refletiram na independência do doente, passando de um grau de Dependência severa no autocuidado, no início da experiência, para um grau de Independência, no final. Resultados semelhantes foram encontrados no estudo de Alcobia, et al (2019), em que foi igualmente aplicado um Programa de Reabilitação baseado nos princípios de Margaret Johnstone, durante 4 meses, num Hospital do Sul do País, com uma amostra de 7 pessoas, tendo-se verificado melhorias na força muscular, equilíbrio corporal, transferências e mobilidade (marcha), que se traduziu em progressos significativos na capacidade funcional, também avaliada através do Índice de Barthel. (20)

Um estudo realizado por Refacho, et al (2019), em que durante 7 semanas foi implementado um treino orientado para a tarefa, revelou também que os ganhos obtidos a nível do equilíbrio e da funcionalidade da marcha se refletiram no aumento da confiança e da autonomia aquando da realização das atividades de vida diária. ${ }^{(21)}$

O risco de queda está fortemente associado ao equilíbrio corporal, por isso, se um Programa de Reabilitação for eficaz na recuperação do equilíbrio corporal, irá também contribuir para a diminuição do risco de queda, o que se verificou neste caso em particular. ${ }^{(20)}$

Constatou-se também que a execução dos exercícios orofaciais permitiu não só diminuir o grau de disfagia do doente, mas também reduzir o nível de paresia facial. No entanto, não se conseguiu reverter totalmente a paresia facial e, embora a articulação de palavras tenha melhorado ao longo do processo, o doente ainda mantinha alguma disartria no final do Programa de Reabilitação.

Um estudo realizado em Évora (2018), assente na implementação de um Programa de Intervenção bastante semelhante ao usado neste Estudo de Caso, que contemplava o posicionamento e mobilização com 
padrão antiespástico, treino de equilíbrio, marcha e força muscular e exercícios de fortalecimento dos músculos da face, revelou melhorias a nível da força muscular, sensibilidade profunda, equilíbrio corporal, marcha e realização das atividades de autocuidado, por parte do doente vítima de AVC, o que corrobora mais uma vez a eficácia deste modelo de Programa de Reabilitação. ${ }^{(15)}$

São bastante mais frequentes os AVC de etiologia hemorrágica $(\mathbf{7 6 , 9 \% )}$ face aos eventos isquémicos $(26,3 \%)$, facto largamente corroborado por inúmeros estudos da literatura atual. No entanto, o AVC isquémico está associado a uma taxa de mortalidade mais baixa e a um melhor tempo de sobrevida inicial, sendo que o AVC hemorrágico representa em média cerca de 1,37 vezes menor probabilidade de sobrevivência. (22)

Relativamente à recuperação funcional pós AVC, esta depende de inúmeros fatores, principalmente da gravidade inicial do AVC, verificando-se geralmente maior gravidade nos casos de hemorragia do que trombose, embora para além da etiologia, a localização e extensão da lesão sejam também fatores preponderantes para o potencial de recuperação. Idades mais jovens, ausência de défices anteriores e um maior comprometimento e participação do doente durante o processo de reabilitação são outros fatores que contribuem para o sucesso da reabilitação. (23)

\section{CONCLUSÃO}

Do relato desta experiência resulta a evidência de que a intervenção do Enfermeiros Especialistas em Enfermagem de Reabilitação traz ganhos específicos, particularmente no equilíbrio, na marcha, no autocuidado e na diminuição do risco de queda.

No entanto, o facto de a paresia facial à direita não ter revertido na totalidade, sugere que pode ser necessário um tempo de intervenção mais longo do que um mês para reverter este défice neurológico.

O tema em estudo é bastante pertinente, devido à elevada incidência de casos de AVC que ainda se verifica na atualidade, causando, na maioria dos casos, uma diminuição acentuada da funcionalidade da pessoa, que se não for devidamente estimulada logo que a situação clínica o permita, poderá resultar em perdas permanentes na sua autonomia.

Esta experiência demonstra que a intervenção em doentes com AVC precisa de ser repensada em termos de gestão de tempo, considerando que neste caso tivemos um contacto de 30 dias e se o doente tivesse um internamento de acordo com a média nacional para doentes com AVC, que é de 12 dias, seria insuficiente, pois teria alta ainda com bastantes défices, o que sugere a necessidade de haver continuidade destes Programas de Reabilitação, por exemplo, no domicílio. (4)

A limitação do estudo é ser o relato de uma experiencia única, contudo foi o tempo de contacto efetivo possível com o caso clínico em análise.
Os resultados obtidos apelam à importância de uma intervenção precoce e contínua no doente após um episódio de AVC, por parte do Enfermeiro Especialista de Enfermagem de Reabilitação, no sentido de contribuir para a recuperação da sua funcionalidade, da sua autonomia nas atividades de vida diárias e, consequentemente para a sua reinserção social enquanto cidadão.

\section{REFERÊNCIAS BIBLIOGRÁFICAS}

1. Assembleia da República. Regulamento das Competências Comuns do Enfermeiro Especialista, n 140/2019, D.R. II Série, N ${ }^{\circ}$ 26. Lisboa: 2019 [citado em 20 julho 2020]. Disponível na Internet:

https: //www.ordemenfermeiros.pt/media/10778/0474404750.p df

2. Ascenção S. Da Qualidade dos Cuidados de Enfermagem à Satisfação das Necessidades do Utente. Porto: 2010 [citado em 20 julho 2020]. Disponível na Internet: https://repositorioaberto.up.pt/bitstream/10216/26626/2/Helena\%20Asceno\%2020 10\%20Dissertao\%20de\%20Mestrado\%20em\%20Cincias\%20de\%20Enf ermagem $\% 20 \% 20$ Da\%20qualidade $\% 20$ dos\%20cuidados\%20\%20satisf ao\%20das\%20neces1.pdf?links=false

3. Roldão L, Conceição F. O Treino de Marcha na Promoção do Autocuidado na Reabilitação da Pessoa com AVC: Revisão Sistemática da Literatura. Projetar Enfermagem - Revista Científica de Enfermagem [revista em linha]. 2019 [citado em 20 julho 2020]; 1: 63-74. Disponível na Internet: https: //projetarenfermagem.pt/wp-

content/uploads/2019/02/Projetar-Enfermagem-n1-2.pdf

4. Direção-Geral da Saúde. Programa Nacional para as Doenças Cérebro-Cardiovasculares. Lisboa: Direção-Geral da Saúde; 2017 [citado em 20 julho 2020]. Disponível na Internet: https://www.dgs.pt/portal-da-estatistica-da-saude/diretoriode-informacao/diretorio-de-informacao/por-serie-882061pdf.aspx?v=\%3d\%3dDwAAAB\%2bLCAAAAAAABAArySzItzVUy81MsTU 1MDAFAHzFEfkPAAAA

5. Organização Mundial da Saúde. Manual STEPS de Acidentes Vascular Cerebrais da OMS: enfoque passo a passo para a vigilância de acidentes vascular cerebrais. Genebra: Organização Mundial da Saúde [citado em 21 julho 2020]. Disponível na Internet:

https: / /www.paho.org/hq/dmdocuments/2009/manualpo.pdf

6. Boumer TC, Firmino TC, Devetak GF, et al. Efeitos do Treino de Marcha com Suporte Parcial de Peso Corporal Associado a Fisioterapia Convencional sobre o Equilíbrio Funcional e a Independência da Marcha Pós-AVC: Estudo Clínico Randomizado. Revista Inspirar Movimento e Saúde [revista em linha]. 2019 [citado em 21 julho 2020]; 19 (4). Disponível na Internet: https: / /www.inspirar.com.br/revista/277287/

7. Margaret Johnstone MCSP. Restauração da Função Motora no Paciente Hemiplégico. 1 ed. São Paulo: Editora Manole LTDA.; 1979.

8. Silveira, MJ. Intervenção do Enfermeiro Especialista em Reabilitação no Doente com Alterações a Nível da Comunicação e Linguagem, em Particular no Doente que Sofreu AVC. Lisboa: 2013 [citado em 21 julho 2020]. Disponível na Internet: https://comum.rcaap.pt/bitstream/10400.26/16199/1/Relat\%c 3\%b3rio\%20\%20Est\%c3\%a1gio\%20M\%c2\%aa\%20Jo\%c3\%a3o.pdf

9. Yin RK. Pesquisa Estudo de Caso - Desenho e Métodos. 2 ed. Porto Alegre: Bookman; 1994.

10. Apóstolo J. Instrumentos para Avaliação em Geriatria (Geriatric Instruments). Coimbra: 2012.

11. Pinto AHVC. Efeitos de um programa de treino combinado de equilíbrio/proprioceção e força muscular no risco de quedas no idoso. Porto: 2018.

12. Barbosa P, Carvalho L, Cruz S. Escala de Quedas de Morse: Manual de utilização. 1 ed. Porto: Escola Superior de Enfermagem do Porto; 2015.

13. Ordem dos Enfermeiros. Instrumentos de recolha de dados para a documentação dos Cuidados Especializados em Enfermagem de Reabilitação. 2016 [citado em 24 julho 2020]. Disponível na 
Internet:

https://www.ordemenfermeiros.pt/arquivo/colegios/Document s/2017/InstRecolhaDadosDocumentacaoCuidEnfReabilitacao_Fin al_2017.pdf

14. Correia T, Sampaio MJ, Almeida R, Garrido C. Paralisia Facial Periférica Diagnóstico, Tratamento e Orientação. Nascer e Crescer [revista em linha]. 2010 [citado em 24 julho 2020]. 19(3): 155-160. Disponível na Internet: http://www.scielo.mec.pt/pdf/nas/v19n3/v19n3a05.pdf

15. Grilo JNF. Melhoria da eficácia das intervenções do enfermeiro especialista de enfermagem de reabilitação na promoção do autocuidado nos doentes com AVC. Évora: 2018 [citado em 24 julho 2020]. Disponível na Internet: https://dspace.uevora.pt/rdpc/handle/10174/23268

16. Simões CMAR, Simões JFFL. Avaliação Inicial de Enfermagem em Linguagem CIPE $®$ segundo as Necessidades Humanas Fundamentais. Referência [revista em linha]. 2007 [citado em 24 julho 2020]. 2(4):9-23. Disponível na Internet: http: / /www.index-f.com/referencia/2007pdf/9-2007-jun.pdf

17. Roche. Calcule o seu IMC: Índice de Massa Corporal [em linha]. [Consult. 24 julho 2020]. Disponível na Internet: https://www.roche.pt/emagrecer/calculadoras/calcimc.cfm

18. International Council of Nurses. Classificação Internacional para a Prática de Enfermagem [em linha] International Council of Nurses; 2019 [atualizado em 2019; Consult. 14 julho 2020]. Disponível na Internet: https://www.icn.ch/what-wedo/projects/ehealth/icnp-browser

19. Araújo F. Ações Autónomas de Enfermagem: Ganhos em Saúde. Porto: 2008 [citado em 26 julho 2020]. Disponível na Internet: https://bdigital.ufp.pt/bitstream/10284/680/1/TESE.pdf

20. Alcobia A, Ferreira R, Soares M, Vieira J. Enfermagem de reabilitação a pessoas idosas com andar comprometido. Journal of Aging \& Innovation [revista em linha]. 2019 [citado em 30 julho 2020]. 8(2):28-43. Disponível na Internet: https: / / repositorio.ipbeja.pt/bitstream/20.500.12207/5252/1/J ०\%C3\%A30\%20Vieira\%20\%20ldosos\%20com\%20Andar\%20Comprometido.pdf

21. Refacho A, Joana $S$, Magalhães $M$, Lourenço $T$, Fernandes $B$. Efeitos do Treino Orientado para a Tarefa na Marcha, Equilíbrio e Medo de Cair Após Acidente Vascular Cerebral: Estudo de Caso Saúde \& Tecnologia: Revista Científica [revista em linha]. 2019 [citado em 30 julho 2020]. 22. Disponível na Internet: https://web.estesl.ipl.pt/ojs/index.php/ST/article/view/2228

22. Figueiredo $A R$, Alexandre $P$, Sónia $M$. Acidente vascular cerebral isquémico vs hemorrágico: taxa de sobrevivência. HIGEIA Revista Científica da Escola Superior de Saúde Dr. Lopes Dias [revista em linha]. 2020 [citado em 14 dezembro 2020]; 3(1):35$45 . \quad$ Disponível na Internet: http://revistahigeia.ipcb.pt/artigos_n3/03_Acidente_vascular_c erebral_isquemico_vs_hemorragico_taxa_de_sobrevivencia.pdf

23. Silva EJA. Reabilitação após o AVC. Porto: 2010 [citado em 14 dezembro 2020]. Disponível na Internet: https://repositorioaberto.up.pt/bitstream/10216/52151/2/Reabilitao\%20aps\%20o\% 20AVC.pdf 